Marc R. Schlaeppi

\title{
Fortbildung
}

Schweiz Z Ganzheitsmed 2014;26:101

DOI: 10.1159/000360336

\section{Viertes Symposium «Integrative Onkologie und Forschung» in St. Gallen}

Zum vierten Mal haben sich am 25. und 26. Oktober 2013 rund 100 Gesundheitsprofessionelle aus der komplementären und konventionellen Medizin am Symposium «Integrative Onkologie und Forschung» (www.integrative-oncology.ch) am Kantonsspital St. Gallen getroffen. Ziel war es zum einen, gemeinsame Forschungsprojekte in der Onkologie $\mathrm{zu}$ initiieren und $\mathrm{zu}$ begleiten, und zum anderen, Fortbildungsreferate und Workshops mit Experten anzubieten. Das Treffen fand erneut im Geiste einer offenen Dialogkultur statt, in deren Rahmen sich Komplementär- und Schulmedizin im Sinne einer Integrativen Medizin begegnen können.

Die Themen der Fortbildungen lauteten unter anderem: «Gedanken zum Leib-Seele-Problem» (Prof. P. Heusser), «Highlights und Trends in der Integrativen Onkologie» (Dr. M. Schlaeppi), «Sinnvoller Einsatz von Phytotherapie» (Dr. J. Hübner), «Heileurythmie als achtsame Bewegungstherapie» (E. Halsmayer), «Inte-

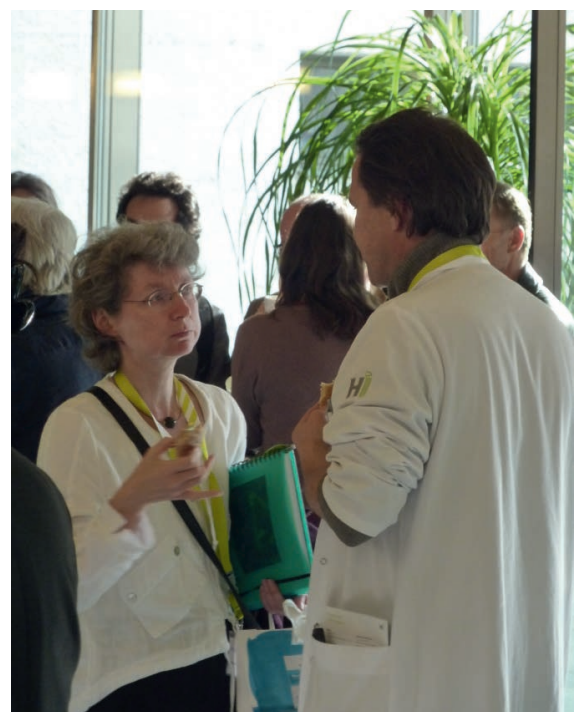

gration von Chinesischer Medizin» (Dr. H. Lampe) und «Was soll ein Curriculum für Integrative Onkologie beinhalten» (Prof. G. Dobos, Dr. P. Voigt, Prof. P. Heusser). Die Referate sind unter www.integrative-oncology. ch/archiv zu finden.

Mitglieder der bestehenden Forschungsgruppen konnten anhand von Postern erste Studienergebnisse vorstellen, insbesondere eine Pilotstudie zur Stressreduktion durch Achtsamkeitstraining von medizinischem Personal, eine retrospektive Analyse von Fiebertherapie durch Mistelinfusionen, ein Review zu äusseren Anwendungen in der Pflege, onkologische Rehabilitation und eine Erarbeitung einer Definition der Integrativen Onkologie durch den Vorstand der European Society for Oncology (ESIO).

In den Projektgruppen wurde weiter an den Themen «Mistel und Fieber», "Achtsamkeitstraining für das Personal», «Postoperative Heileurythmie für Patientinnen mit Brustkrebs», «Bewusstseinsraum - Integratives Board» sowie «Äussere Anwendungen bei Schlafstörungen auf einer Akutstation» gearbeitet. Bei einigen der Forschungsprojekte dürften schon im Jahr 2014/2015 weitere Ergebnisse erwartet werden (Mistel und Fieber, Bewusstseinsraum, Review über äussere Anwendungen), während sich bei den anderen Forschungsprojekten (Achtsamkeitstraining, Heileurythmie, Schlafstörungen und äussere Anwendungen) die Protokolle noch in der Entstehung befinden.

Die fünf Projektgruppen werden sich am 31. Oktober 2014 erneut in St. Gallen treffen, um ihre Arbeit fortzuführen. Wer Interesse hat, sich

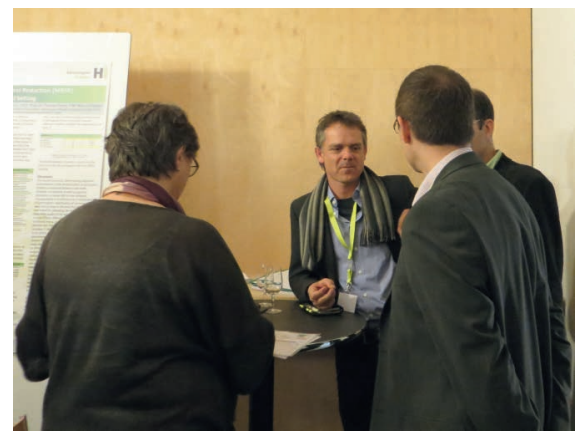

an der einen oder anderen Projektgruppe (www.integrative-oncology.ch/ Projektgruppen) aktiv zu beteiligen, ist herzlich eingeladen, sich bei Frau Ermina Zecic (ermina.zecic@kssg.ch), Zentrum für Integrative Medizin, Kantonsspital St. Gallen, zu melden.

Das Symposium stand unter der Leitung des Kantonsspitals St. Gallen (Prof. T. Cerny, Dr. M. Schlaeppi), der Universität Witten/Herdecke (Prof. P. Heusser) und der Universität Bern (PD Dr. U. Wolf). Partnerorganisationen waren die Krebsliga Schweiz (KLS) und die Arbeitsgemeinschaft Prävention und Integrative Onkologie (PRIO) der Deutschen Krebsgesellschaft. Das Symposium konnte auch durch die freundlichen Förderungen der «Günter und Regine Kelm Stiftung» sowie verschiedener Firmen ermöglicht werden. Diese sind unter www.integrative-oncology.ch/ sponsoren zu finden.

Das fünfte «Symposium Integrative Onkologie und Forschung» wird am 30. und 31. Oktober 2015 in St. Gallen stattfinden. Hauptthema wird die «Integrative Kinderonkologie» sein. Die Projektgruppen werden wieder die Möglichkeit haben, sich zu treffen, Forschungsergebnisse vorzustellen und die nächsten Schritte zu erarbeiten.
Dr. med. Marc R. Schlaeppi, MSc

Zentrum für Integrative Medizin, Kantonsspital St. Gallen

Greithstrasse 20, 9007 St. Gallen, Schweiz

marc.schlaeppi@kssg.ch 